\title{
A rare case of metastatic esthesioneuroblastoma
}

\author{
Emilia Marciuc ${ }^{1,2}$, M. Barcan1, S. Popa ${ }^{1}$, B.I. Dobrovățt ${ }^{1,2}$, \\ R.M. Popescu ${ }^{1,2}$, R. Buga ${ }^{1,2}$, D. Haba ${ }^{1,2}$ \\ 1 Department of Radiology and Medical Imaging of Emergency \\ Hospital "Prof. Dr. N. Oblu", Iasi, RomanIA \\ 2 "Grigore T. Popa" University of Medicine and Pharmacy, lasi, \\ ROMANIA
}

\begin{abstract}
Olfactory neuroblastoma, also known as esthesioneuroblastoma (ENB), is a rare malignant tumour of the basal layer of the olfactory epithelium, which originally develops unilaterally, accounting for 3-6\% of all intranasal tumours. We present the case of a patient with a voluminous ethmoidal lesion that invaded the left basal frontal lobe and left orbit. The biopsy revealed a stage C KADISH, grade III neuroblastoma. The patient followed a multimodal treatment with chemotherapy and radiation therapy to which he responded partially, then returned after 11 months for sphincter disorder and bilateral sciatic type pain. An MRI showed metastasis of the filum terminale, the anatomopathological exam identifying also neuroblastoma. $\mathrm{CT}$ and MRI imaging are required for a correct assessment of the regional extension of olfactory neuroblastoma, response to oncological treatment but also for the detection of secondary lesions found in a small number of cases.
\end{abstract}

\section{INTRODUCTION}

ENB is a rare malignancy, includes $3 \%$ of all intranasal tumours (1), was first described in 1924 (2), and it develops from the olfactory epithelium of the cribriform plate. These tumours are initially located unilaterally, and then invades orbit, nasal fossa, skull base, and intracranial space. The risk factors are unknown and there is a bimodal incidence at 10-15 years and 40-50 years. The most common symptoms are nasal congestion, anosmia, epistaxis, headache, and diplopia. These tumours have a high potential for regional and distance extension and multimodal treatment may help patients and increase their survival rate (3).

\section{CASE PRESENTATION}

A 33-year-old patient presented to the emergency room with headache and vomiting. CT exploration was recommended which showed hydrocephalus and a lesion that occupied the ethmoidal air cells, an extension in the nasal fossa, the anterior cranial fossa with bone lysis. A gadolinium enhanced MRI was performed that showed a 33/34/23

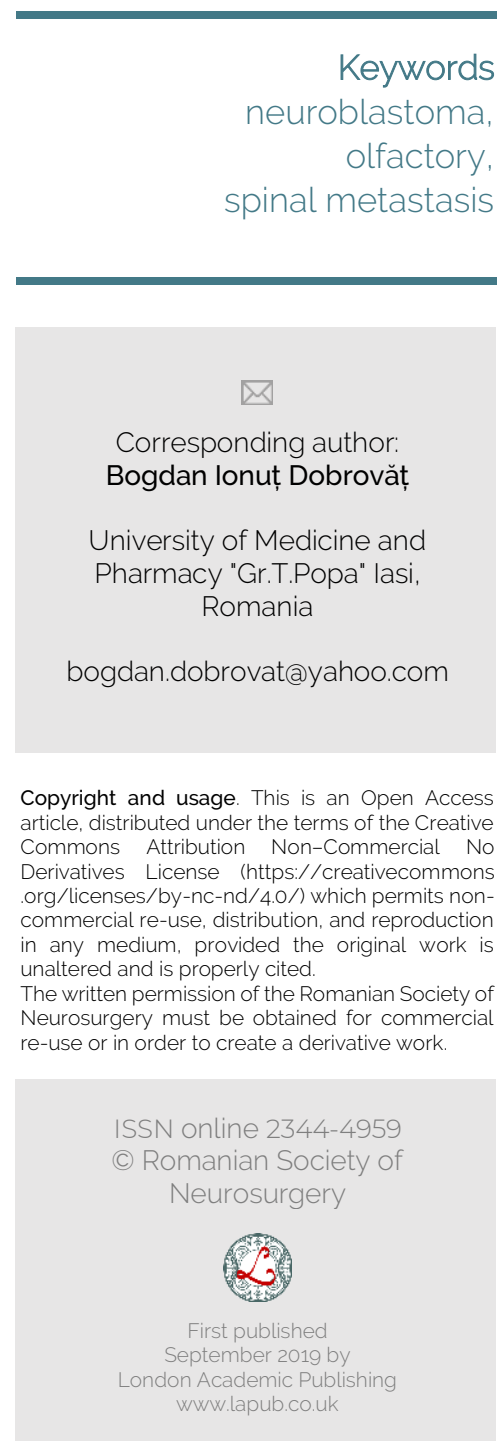


$\mathrm{mm}$ (AP/CC/T) lesion, with a heterogeneous enhancement, with calcified areas, occupying mostly of the nasal fossa and all ethmoid cells, most likely developing from the cribriform plate, invading the orbit (with mass effect on the internal muscles) and frontal lobes, more evident in the left side (Fig. 1, Fig. 2, Fig. 3, Fig. 4).

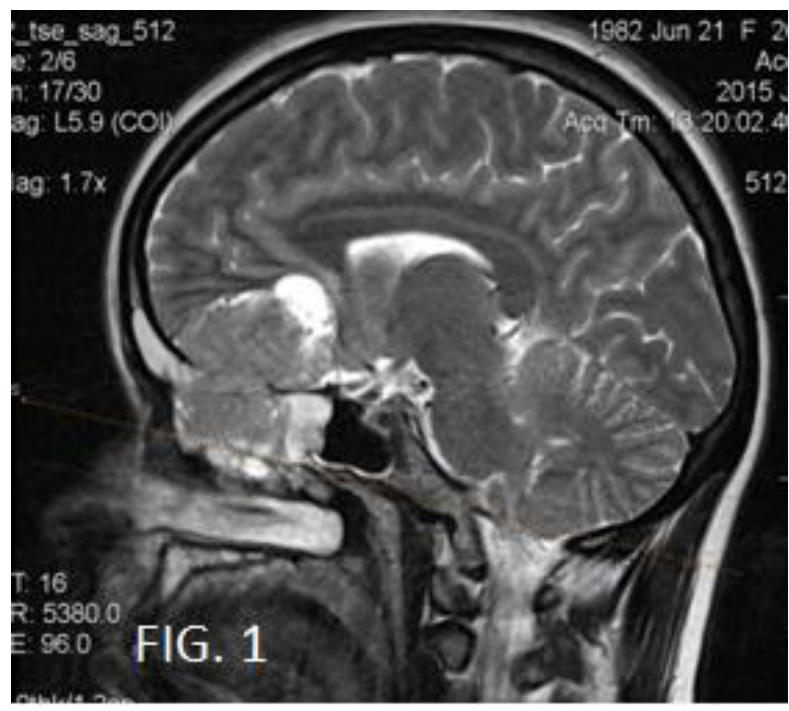

Figure 1. Sag T2WI. The tumour occupying the nasal fosa, originating from cribriform blade with superior extension.

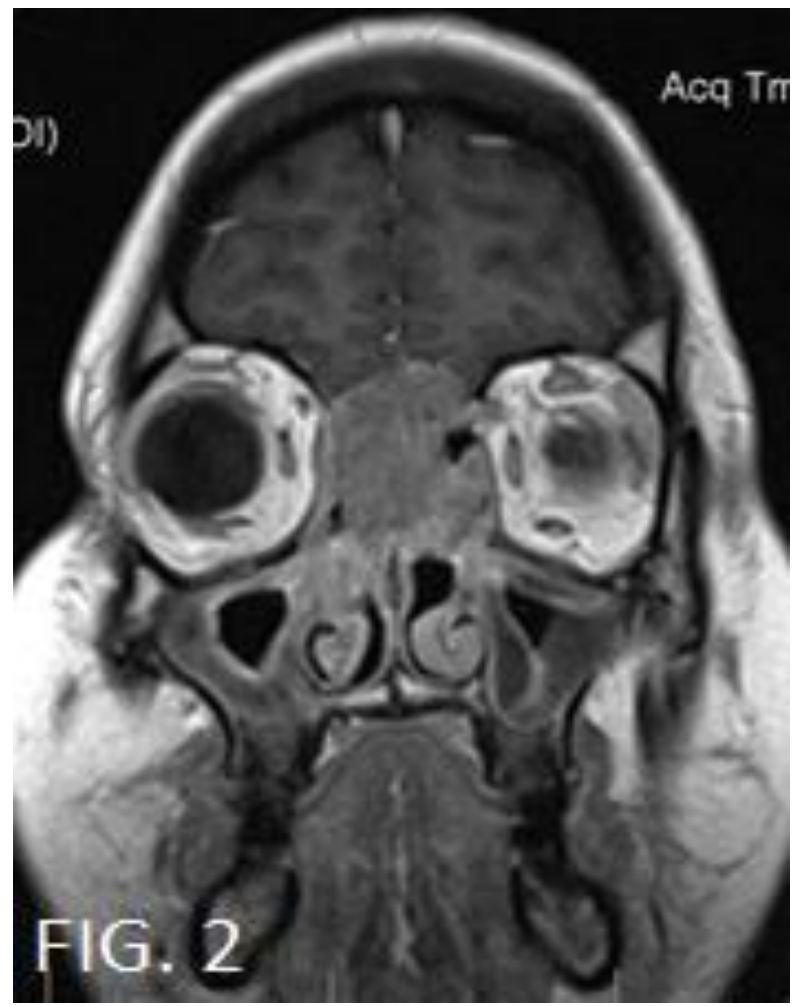

FIGURE 2. MRI Cor Gd-T1WI.

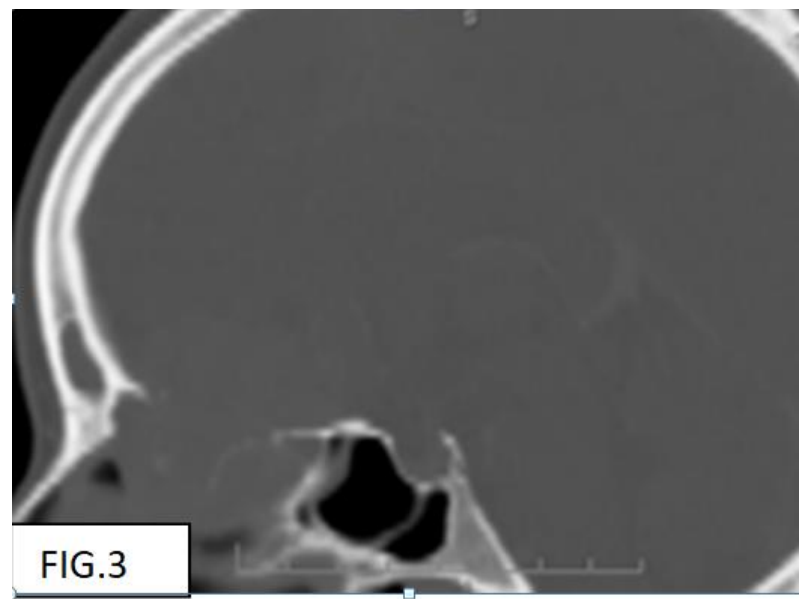

FIGURE 3. Sag CT (bone window): bone lysis.
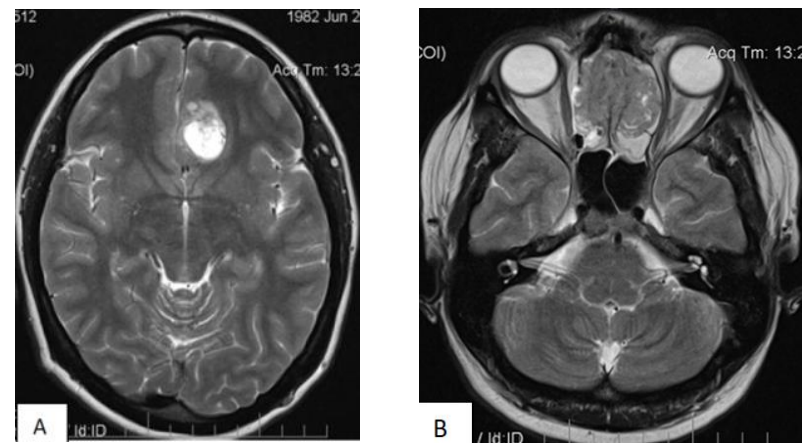

FIGURE 4. (A) Axial T2Wl-extension of the frontal lobe; (B) Axial T2WI - mass effect on the internal muscles in the left orbit.

After the biopsy, the anatomopathological exam concluded stage III Kadish group C neuroblastoma. In this case the patient followed two chemotherapy cycles but abandoned it later because he was known to have hepatitis $\mathrm{B}$ and the viremia level was increasing. Therefore, he continued only with radiotherapy.

The patient returned to control after six months when the MRI scan showed a decrease in tumour size, with the same characteristics as before, and was kept under control for one year. After one year the patient came back with bilateral sciatica and major sphincter dysfunctions. The MRI exam of lumbar region highlighted a hyperintense $\mathrm{T} 1$ and $\mathrm{T} 2$, hypointense STIR lesion in the S1 vertebral body and a nodular vivid enhancing lesions of cauda equina in S1 and S2 region, suggestive of secondary lesion (Fig. 5, Fig. 6); the anatomopathological examination confirmed the diagnosis of metastasis from neuroblastoma. 


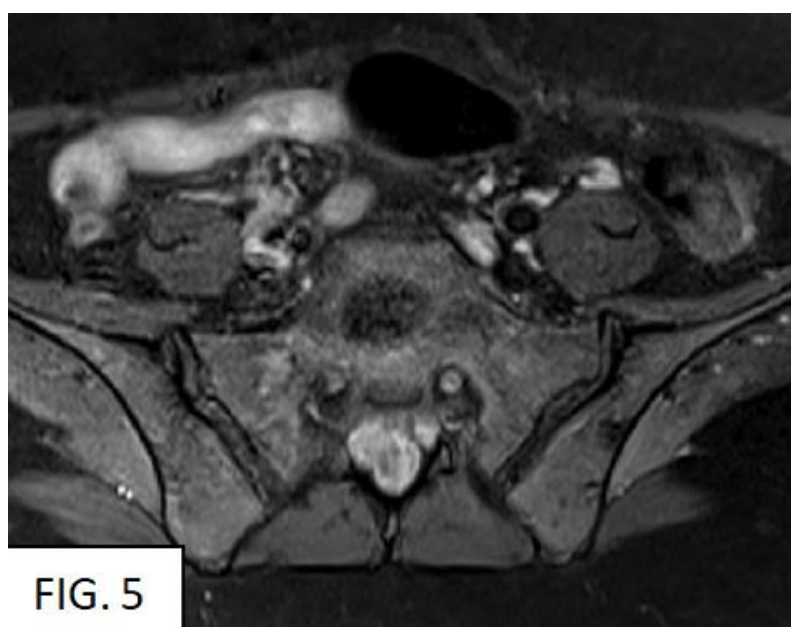

FIGURE 5. MRI Axial STIR - S1 vertebral body level.
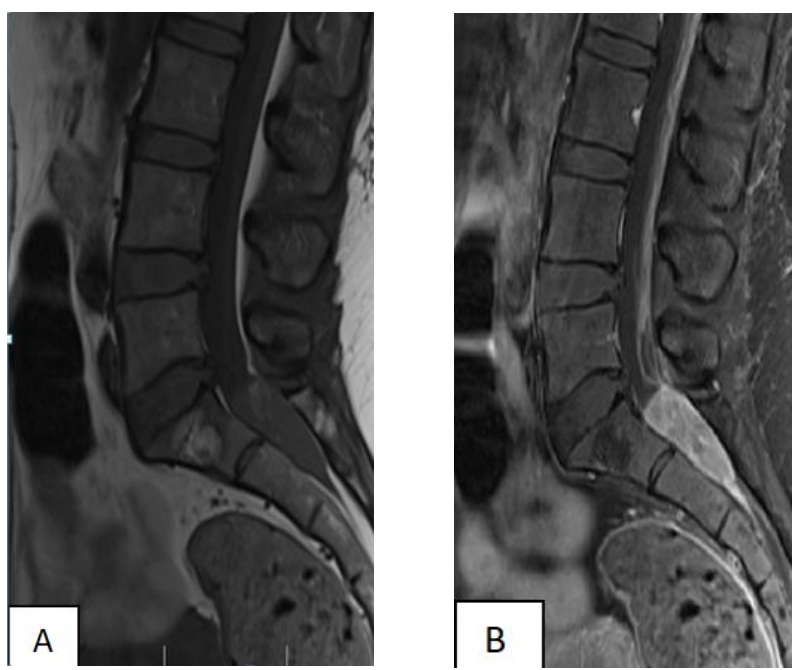

FIGURE 6. S1 secondary lesion (A - T1Wl; B - Gd enhanced-T1WI))

After 10 episodes of lumbar radiotherapy, the patient returned accusing headache, diplopia and diffuse cervico-lumbar pain. MRI exploration revealed a stationary aspect of the tumor compared to the previous exam. The patient wanted to be discharged and unfortunately, he died shortly afterwards.

\section{DisCUSSIONS}

Olfactory neuroblastoma is a tumour which originates from the olfactory cells of the cribriform blade and, although it develops initially in an unilateral manner, it invades afterwards the bilateral intracranial spaces. Kadish classifies these tumours in three classes (4): A - limited to the nasal cavity, B infiltrated into the nasal cavity and paranasal and $C$ - extended beyond the nasal and paranasal cavity. Our case was classified in stage $C$ and histologically in class III. There are four histological classes that divide this type of tumour by histological cellularity and histochemical analysis. According to some authors, ENB it is most likely congenital, and it develops even in intrauterine life (5).

The role of imaging in the staging of ENB is very important. In $40 \%$ of cases, it extends in the paranasal sinuses, in 30\% extension is intracranial, and in 30\% extension is orbital (6). MRI is the most used technique in staging because the report on the extension of the tumour in the adjacent tissues can be very thorough.

Very important are the CECT and PET-CT exams of lymph nodes because esthesioneuroblastoma disseminates in cervical and laterocervical lymph nodes which are asymptomatic and undetectable on physical exam. CECT and PET-CT can highlight these lymph nodes by marked contrast enhancement on CECT and moderate to highly avid of FDG on PET/CT.

Some studies highlight a cervical dissemination in the lymph nodes in $30 \%$ of cases and the presence of secondary lesions are rare and very rare for spine (7). In our case there was secondary involvement of vertebral body of $\mathrm{S} 1$ and also a metastasis of cauda equina in $\mathrm{S} 1$ and $\mathrm{S} 2$ region.

Many studies show that patients treated with surgery have a 5 -year survival rate of about $50 \%$, while surgery combined with chemotherapy prolong survival to $65 \%$ at 5 years in the case of local esthesioneuroblastoma $(8,9)$.

In local advanced ENB, resection of the cervical lymph nodes is performed to reduce the risk of recurrences. Postoperative chemotherapy and radiotherapy in patient with lymph nodes metastasis have decreased recurrences and the survival rate at 10 years was $80 \%$.

Chemotherapy combined with radiotherapy is widely accepted recently, with a survival rate of $35 \%$. Despite lymph node resection, radiotherapy and chemotherapy, in most cases of advanced ENB lymph node recurrence is detected 12 months after completion of treatment.

Currently, radiotherapy in ENB is used in combination with surgery and chemotherapy because there are no randomized trials to guide radiotherapy treatment, but radiotherapy plays an important role in the treatment plan in early stages with definitive cure and good tumour control. The 
doses used are 50-60 Gy with intensity-modulated radiation therapy to minimize doses to the optic structures, pituitary gland and brain (10).

\section{CONCLUSIONS}

Olfactory neuroblastoma is a rare tumour that originates from olfactory epithelium and extends into the orbit, nasal fossa, skull base and intracranial space. The combined use of CT and MRI techniques is excellent in providing necessary information for treatment planning. Recognizing the limitation of current data, surgical resection and postoperative radiation therapy for resectable lesions is the most commonly used management approach. For unresectable tumours, radiation alone or chemoradiation therapy are also viable treatment options. Palliative chemotherapy has not shown a clear survival benefit in patients with recurrent and/or metastatic disease.

Considering the metastatic potential of this type of tumour, a rigorous imaging examination is required for an early diagnosis and a more effective treatment.

\section{REFERENCES}

1. Berger L, Luc R, Richard D. L'esthesioneuroepitheliome olfactif. Bull Assoc Fr Etude Cancer 1924; 13: 410-421.

2. Kumar M, Fallon RJ, Hill JS, Davis MM.
Esthesioneuroblastoma in children. J Pediatr Hematol Oncol 2002; 24: 482-487.

3. Chao KS, Kaplan C, Simpson JR, et al. Esthesioneuroblastoma: the impact of treatment modality. Head Neck 2001.

4. Kadish S, Goodman M, Wang CC. Olfactory neuroblastoma: a clinical analysis of 17 cases. Cancer.1976;37(3):1571-6.

5. Thompson LD. Olfactory neuroblastoma. Head Neck Pathol. 2009;3(3):252-259. doi:10.1007/s12105-009-01252.

6. Morita A, Ebersold MJ, Olsen KD, Foote RL, Lewis JE, Quast LM. Esthesioneuroblastoma: prognosis and management. Neurosurgery 1993; 32: 706-714; discussion 714-715.

7. Shirzadi AS, Drazin DG, Strickland AS, Bannykh SI, Johnson JP. Vertebral column metastases from an esthesioneuroblastoma: chemothera- py, radiation, and resection for recurrence with 15-year followup. Case Rep Surg 2013; 2013: 107315.

8. Yan-Feng Chen, An-Kui Yang, Quan Zhang, Dian Ouyang, Wen-Kuan Chen and Fu-jin Chen. Clinical analysis of 53 cases of esthesioneuroblastoma; Chinese Journal of Cancer 2009; 28:3, 259-262.

9. Gupta S, Husain N, Sundar S. Esthesioneuroblastoma chemotherapy and radiotherapy for extensive disease: a case report. World J Surg Oncol. 2011;9:118. Published 2011 Oct 5. doi:10.1186/1477-7819-9-118.

10. Patel SG, Singh B, Stambuk HE, et al. Craniofacial surgery for esthesioneuroblastoma: report of an international collaborative study. J Neurol Surg B Skull Base. 2012;73(3):208-220. doi:10.1055/s-0032-1311754. 